\title{
1 Method for culturing Candidatus Ornithobacterium hominis
}

2 Authors:

3 Katrina A Lawrence ${ }^{1}$, Tegan M Harris ${ }^{1}$, Susannah J Salter ${ }^{2}$, Rick W Hall ${ }^{1}$, Heidi C Smith-

4 Vaughan $^{1}$, Anne B Chang ${ }^{1,3}$, Robyn L Marsh ${ }^{1 \#}$

5

6 Affiliations:

7 1. Menzies School of Health Research, Charles Darwin University, Darwin, Australia

8 2. Pathogen Genomics, Wellcome Sanger Institute, Genome Campus, Hinxton, UK

9 3. Department of Respiratory and Sleep Medicine, Queensland Children's Hospital and the

10 Centre for Children's Health Research, Queensland University of Technology, Brisbane,

11 Australia

12

$13 \quad$ "Corresponding author:

14 Dr Robyn Marsh

15 Email: robyn.marsh@menzies.edu.au

16 Address:

17 Menzies School of Health Research

$18 \quad$ PO Box 41096

19 Casuarina, NT, Australia, 0811

20

21 Keywords:

22 Candidatus Ornithobacterium hominis, culture, nasopharyngeal 


\section{Abstract}

24 Candidatus Ornithobacterium hominis has been detected in nasopharyngeal microbiota sequence data from around the world. This report provides the first description of culture conditions for isolating this bacterium. The availability of an easily reproducible culture method is expected to facilitate deeper understanding of the clinical significance of this species.

\section{Manuscript text}

Candidatus Ornithobacterium hominis $(\mathrm{OH})$ is a bacterium that has been detected in nasopharyngeal microbiota sequence data from around the world but has never been cultured (Salter et al., 2019; Salter et al., 2017). This bacterium is of growing interest as polymerase chain reaction (PCR)-based studies found that $\mathrm{OH}$ was prevalent and persistent in the nasopharynx of a paediatric population at high-risk of respiratory infection (Salter et al., 2019; Salter et al., 2017). Additionally, the closest known relative of $\mathrm{OH}$ is Ornithobacterium rhinotracheale; a respiratory pathogen of birds (Zahra et al., 2013). These observations prompt research to understand the pathogenic potential and clinical significance of $\mathrm{OH}$.

Although genomes can be derived from metagenomic data, $\mathrm{OH}$ isolates are needed to deepen understanding of the bacterium's role in human respiratory infections. The aim of this study was to determine culture conditions for recovery of $\mathrm{OH}$ isolates.

The study was approved by the Human Research Ethics Committee of the Northern Territory Department of Health and Menzies School of Health Research (Approval number: 0785). Culture was performed using biobanked nasopharyngeal swabs that were collected from four Australian children (age 1-2 years) immediately prior to bronchoscopy for investigation for chronic suppurative lung disease (Marsh et al., 2016). The swabs had been stored in skim 
milk-tryptone-glucose-glycerol broth (STGGB) at $-80^{\circ} \mathrm{C}$ for up to 10 years and had two freeze-thaw cycles prior to $\mathrm{OH}$ culture. These swabs were selected as all were $\mathrm{OH}-$ positive by $16 \mathrm{~S}$ rRNA gene sequencing at 5-55\% relative abundance (Marsh et al., 2016).

Ten microlitres of the STGGB swab media was inoculated onto Tryptic Soy Agar with 5\% Sheep Blood (TSA), Horse Blood Columbia agar (HBA), Chocolate agar and Brain Heart Infusion agar (BHI). The plates were incubated aerobically, microaerophillically (Campygen, Oxoid) and anaerobically (Anaerogen, Oxoid) at $35^{\circ} \mathrm{C}$ for up to five days. Aerobic culture was also performed in the presence of a wet sponge to provide increased humidity (Mayahi et al., 2016). Oxidase testing was done using oxidase test strips (Oxoid). Tributyrin hydrolysis was determined using Catarrhalis discs (Remel). Production of $\beta$-lactamase was determined using nitrocefin (Oxoid).

60

Primary cultures were reviewed for colonies resembling O. rhinotracheale (van Empel and Hafez, 1999). Colonies of oxidase-positive, Gram-negative pleomorphic bacilli were screened using PCR targeting OH-specific regions of the 16S rRNA and toxA genes, as described previously (Salter et al., 2019). PCR-positive isolates were confirmed using genome sequencing. Genomes were assembled de novo using the Microbial Genome Assembly Pipeline (MGAP) v1.0 (https://github.com/dsarov/MGAP---Microbial-Genome-

67 Assembler-Pipeline) (Chapple et al., 2016). OH identification was confirmed where isolates had $>96 \%$ average nucleotide identity (Kim et al., 2014; Richter and Rossello-Mora, 2009) when compared to draft $\mathrm{OH}$ genomes $\mathrm{OH}-22767$ (GenBank accession NZ_UNSC00000000.1) and OH-22803 (GenBank accession UNSD00000000.1). Both draft genomes were derived from metagenomic analysis of nasopharyngeal swabs from Thai children (Salter et al., 2019) . Isolate genomes were mapped against the draft $\mathrm{OH}$ genomes 
using the Synergised Pipeline for Analysis of Next Generation Sequencing Data in Linux (SPANDx) v3.2.1 (Sarovich and Price, 2014), which wraps Burrows-Wheeler Aligner (Li and Durbin, 2009), Sequence Alignment/Map (SAM) tools (Li et al., 2009), Picard Tools and Genome Analysis Tool Kit (McKenna et al., 2010). Genomes were aligned using draft $\mathrm{OH}$ genome $\mathrm{OH}-22803$ as the reference with an $O$. rhinotracheale genome (ORT-UMN 88,

GenBank accession CP006828.1) included as an outgroup. Maximum parsimony phylogenomic trees were generated using Phylogenetic Analysis Using Parsimony (PAUP) v4.0a153 (Swofford, 1998) and visualised using FigTree replicates. Lipopolysaccharide comparisons were generated using Easyfig (Sullivan et al., 2011). The $\mathrm{OH}$ isolate genomes are available from the Sequence Read Archive (SRA; BioProject number: PRJNA510696).

$\mathrm{OH}$ was successfully cultured from all four swabs. Primary isolation was challenging due to substantial overgrowth by other taxa (Figure 1). Of the conditions tested, optimal primary culture was achieved using TSA incubated in a microaerophilic atmosphere at $35^{\circ} \mathrm{C}$ for up to five days. $\mathrm{OH}$ also grew on $\mathrm{HBA}$, Chocolate agar and $\mathrm{BHI}$; however, isolates were not consistently recovered from these media. Aerobic growth was possible but required additional humidity (e.g. incubating plates in a box containing a wet sponge).

93 Under microaerophilic conditions, $\mathrm{OH}$ colonies were pleomorphic, glistening, grey and 94 concave. Colonies ranged in size from 1-3 mm after 48-120 hours incubation. All isolates were pleomorphic Gram-negative bacilli. Consistent with the phenotype predicted by the draft genomes (Salter et al., 2019), OH isolates were oxidase-positive, catalase-negative and

97 all produced $\beta$-lactamase. All isolates also hydrolysed tributyrin. Some pure isolates 
produced two colony morphologies (Figure 1D). This phenotype is suggestive of small-cell variants (Zahra et al., 2013) as both colony types were positive by $\mathrm{OH}$ 16S rRNA and toxA PCR.

The $\mathrm{OH}$ isolate genomes had average nucleotide identity of $97.86-98.23 \%$ with draft genomes $\mathrm{OH}-22803$ and $\mathrm{OH}-22767$, indicating that they are members of the same species. Phylogenomic analysis demonstrating the high similarity between the Australian isolates and draft $\mathrm{OH}$ genomes from Thailand is shown in Figure 2. All isolate genomes contained distinct lipopolysaccharide (LPS) biosynthesis clusters which differed to those of the draft genomes (Figure 3). $\beta$-lactamase production was associated with mobile genetic elements that were different in each isolate and occurred at different loci. All isolates also had genes encoding efflux pumps associated with multi-drug resistance.

In summary, following identification of $\mathrm{OH}$ in silico, we now report culture conditions for its propagation. Of the conditions tested, optimal growth was achieved using TSA with incubation for up to five days in a microaerophilic atmosphere; conditions which are not part of standard culture used to recover respiratory pathogens from nasopharyngeal swabs (Satzke et al., 2013). Primary isolation was challenging due to extensive overgrowth by other flora. We recommend OH-specific PCRs (Salter et al., 2019) are used to confirm isolate identity. The $\mathrm{OH}$ colonial morphology was similar to that reported previously for $O$. rhinotracheale (van Empel and Hafez, 1999), including growth of multiple colony morphologies suggestive of small-cell variants (Zahra et al., 2013). The significance of this observation is unknown; however, small-cell variants of other bacteria (e.g. Staphylococcus aureus) have been associated with poorer clinical outcomes in patients with respiratory disease (Wolter et al., 2013). Association of $\beta$-lactamase genes with multiple mobile genetic elements indicates that 
$123 \mathrm{OH} \beta$-lactam resistance has been acquired through several independent events. Heterogeneity

124 among the LPS cluster is suggestive of multiple capsular types, consistent with observations

125 from earlier DNA-based studies (Salter et al., 2019; Salter et al., 2017). The availability of an

126 easily reproducible culture method is expected to facilitate deeper understanding of the

127 clinical significance of $\mathrm{OH}$. 
References

129

130

Chapple, S.N., Sarovich, D.S., Holden, M.T., Peacock, S.J., Buller, N., Golledge, C., Mayo, M., Currie, B.J., Price, E.P., 2016. Whole-genome sequencing of a quarter-century melioidosis outbreak in temperate Australia uncovers a region of low-prevalence endemicity. Microb Genom, 2, e000067.

Kim, M., Oh, H.S., Park, S.C., Chun, J., 2014. Towards a taxonomic coherence between average nucleotide identity and 16S rRNA gene sequence similarity for species demarcation of prokaryotes. Int J Syst Evol Microbiol, 64, 346-51.

Li, H., Durbin, R., 2009. Fast and accurate short read alignment with Burrows-Wheeler transform. Bioinformatics, 25, 1754-60.

Li, H., Handsaker, B., Wysoker, A., Fennell, T., Ruan, J., Homer, N., Marth, G., Abecasis, G., Durbin, R., Genome Project Data Processing, S., 2009. The Sequence Alignment/Map format and SAMtools. Bioinformatics, 25, 2078-9.

Marsh, R.L., Kaestli, M., Chang, A.B., Binks, M.J., Pope, C.E., Hoffman, L.R., SmithVaughan, H.C., 2016. The microbiota in bronchoalveolar lavage from young children with chronic lung disease includes taxa present in both the oropharynx and nasopharynx. Microbiome, 4, 37.

Mayahi, M., Gharibi, D., Ghadimipour, R., Talazadeh, F., 2016. Isolation, identification and antimicrobial sensitivity of Ornithobacterium rhinotracheale in broilers chicken flocks of Khuzestan, Iran. Vet Res Forum, 7, 341-346.

Mckenna, A., Hanna, M., Banks, E., Sivachenko, A., Cibulskis, K., Kernytsky, A., Garimella, K., Altshuler, D., Gabriel, S., Daly, M., Depristo, M.A., 2010. The Genome Analysis Toolkit: a MapReduce framework for analyzing next-generation DNA sequencing data. Genome Res, 20, 1297-303. 
152 Richter, M., Rossello-Mora, R., 2009. Shifting the genomic gold standard for the prokaryotic species definition. Proc Natl Acad Sci U S A, 106, 19126-31.

Salter, S.J., Scott, P., Page, A.J., Tracey, A., De Goffau, M.C., Cormie, C., Ochoa-Montano, B., Ling, C.L., Tangmanakit, J., Turner, P., Parkhill, J., 2019. Candidatus Orthinobacterium hominis sp.: insights gained from draft genomes obtained from nasopharyngeal swabs. Microb Genom, 5.

Salter, S.J., Turner, C., Watthanaworawit, W., De Goffau, M.C., Wagner, J., Parkhill, J.,

Sarovich, D.S., Price, E.P., 2014. SPANDx: a genomics pipeline for comparative analysis of

Satzke, C., Turner, P., Virolainen-Julkunen, A., Adrian, P.V., Antonio, M., Hare, K.M.,

Swofford, D.L. 1998. PAUP*: Phylogenetic Analysis Using Parsimony (*and Other Methods) 4ed. Sunderland, Massachusetts: Sinauer Associates.

Sullivan, M.J., Petty, N.K., Beatson, S.A., 2011. Easyfig: a genome comparison visualizer. Bioinformatics, 27, 1009-10. Pathol, 28, 217-27. 
Wolter, D.J., Emerson, J.C., Mcnamara, S., Buccat, A.M., Qin, X., Cochrane, E., Houston, L.S., Rogers, G.B., Marsh, P., Prehar, K., Pope, C.E., Blackledge, M., Deziel, E., Bruce, K.D., Ramsey, B.W., Gibson, R.L., Burns, J.L., Hoffman, L.R., 2013. Staphylococcus aureus small-colony variants are independently associated with worse lung disease in children with cystic fibrosis. Clin Infect Dis, 57, 384-91.

Zahra, M., Ferreri, M., Alkasir, R., Yin, J., Han, B., Su, J., 2013. Isolation and characterization of small-colony variants of Ornithobacterium rhinotracheale. J Clin Microbiol, 51, 3228-36.

\section{Figure 1: $\mathrm{Ca}$. Ornithobacterium hominis colony morphology}

A) Primary isolation of $\mathrm{Ca}$. O. hominis isolate $903 \mathrm{C} 1$ on TSA after 120 hours aerobic incubation in the presence of a wet sponge. Arrow indicates a $\mathrm{Ca}$. O. hominis colony.

B) Purified $\mathrm{Ca}$. O. hominis isolate $903 \mathrm{C} 1$ after 120 hours microaerophillic incubation on TSA. Pure culture of this strain produced a uniform colony size.

C) Primary isolation of $\mathrm{Ca}$. O. hominis isolate $902 \mathrm{C} 1$ on TSA after 120 hours microaerophillic incubation. Arrow indicates a $\mathrm{Ca}$. O. hominis colony.

D) Purified $\mathrm{Ca}$. O. hominis isolate $902 \mathrm{C} 1$ after 120 hours microaerophillic incubation on TSA. Pure culture of this isolate producted two colony morphotypes.

\section{Figure 2: Phylogenomic analysis of the $\mathrm{Ca}$. Ornithobacterium hominis isolates}

A midpoint-rooted maximum parsimony tree was constructed based on 764 biallelic single nucleotide polymorphisms (SNPs) orthologous to the four Australian $\mathrm{Ca}$. O. hominis isolates (900C2, 902C1, 903C1 and 916C1); two previously reported draft $\mathrm{Ca}$. O. hominis genomes from Thailand (OH-22767 and OH-22803); and an O. rhinotracheale outgroup (ORT-UMN 88). Bar indicates a distance of 50 SNPs. 
202 Figure 3: Comparison of $\mathrm{Ca}$. Ornithobacterium hominis lipopolysaccharide biosynthesis

203 loci.

204 A tblastx alignment of the lipopolysaccharide biosynthesis clusters in the four Australian $\mathrm{OH}$

205 isolates compared to draft genomes $\mathrm{OH}-22767$ and $\mathrm{OH}-22803$ derived from Thailand(2).

206

207 Acknowledgements

208 We acknowledge the support of all families that participated in this study. We are also

209 grateful to Harry Owen from Menzies School of Health Research for his technical assistance.

210 The study was supported by funding from the Centre for Research Excellence (CRE) in Ear

211 and Hearing Health of Aboriginal and Torres Strait Islander Children (NHMRC

212 APP1078557). RLM and HSV are supported by post-doctoral fellowships from the CRE in

213 Respiratory Health for Aboriginal and Torres Strait Islander Children (NHMRC 1040830).

214 The views expressed in this publication are those of the authors and do not reflect the views

215 of the NHMRC. SJS was supported by the Wellcome Trust (Grant Number 098051). 